Article

\title{
Experimental and Numerical Investigation of Termination Impedance Effects in Wireless Power Transfer via Metamaterial
}

Giovanni Puccetti ${ }^{1, *}$, Christopher J. Stevens ${ }^{2}$, Ugo Reggiani ${ }^{1}$ and Leonardo Sandrolini ${ }^{1}$

${ }^{1}$ Department of Electrical, Electronic, and Information Engineering "Guglielmo Marconi”-DEI, University of Bologna, Viale Risorgimento 2, I-40136 Bologna, Italy;

E-Mails: ugo.reggiani@unibo.it (U.R.); leonardo.sandrolini@unibo.it (L.S.)

${ }^{2}$ Department of Engineering Science, University of Oxford, Parks Road, Oxford OX1 3PJ, UK;

E-Mail: chris.stevens@eng.ox.ac.uk

* Author to whom correspondence should be addressed; E-Mail: giovanni.puccetti2@unibo.it; Tel.: +39-051-2093578.

Academic Editor: K. T. Chau

Received: 8 December 2014 / Accepted: 26 February 2015 / Published: 9 March 2015

\begin{abstract}
This paper presents an investigation of the transmitted power in a wireless power transfer system that employs a metamaterial. Metamaterials are a good means to transfer power wirelessly, as they are composed of multiple inductively-coupled resonators. The system can be designed and matched simply through magneto-inductive wave theory, particularly when the receiver inductor is located at the end of the metamaterial line. However, the power distribution changes significantly in terms of transmitted power, efficiency and frequency if the receiver inductor slides along the line. In this paper, the power distribution and transfer efficiency are analysed, studying the effects of a termination impedance in the last cell of the metamaterial and improving the system performance for the resonant frequency and for any position of the receiver inductor. Furthermore, a numerical characterisation is presented in order to support experimental tests and to predict the performance of a metamaterial composed of spiral inductor cells with very good accuracy.
\end{abstract}

Keywords: wireless power transfer; metamaterial; magneto-inductive waves; resonator 


\section{Introduction}

In recent years, wireless power transfer (WPT) has been one of the most interesting and innovative topics studied by both research centres and technology industries, particularly due to the large number of potential applications in which it can be applied (vehicle charging, electronic and biomedical devices [1-3]). However, even if WPT is widely developed for electronic devices and improved for simple systems, to date, there are still a lot of open issues, e.g., the low efficiency due to large distances or misalignments between emitter and receiver coils. Generally, resonators or metamaterials can be used in order to solve these problems [4], arranging, for example, the coupled resonators along a line or in two directions in a plane and letting the receiver slide on them or in a slab whose electrical parameters can be optimised in order to enhance the power transfer efficiency between two-faced coils [5,6]. In this way, power transfer for longer distances is enabled. Such a multiresonator system introduces new problems, particularly in terms of efficiency and frequency: in fact, for a large number of coupled resonators, the efficiency falls, and the power delivered to the receiver coil no longer coincides with the maximum transmitted one for every operating frequency.

In a previous paper, Stevens showed that the efficiency can be improved along a metamaterial line, as depicted in Figure 1, with the introduction of a termination impedance connected to the last cell, whose value depends on the receiver position [7]. This paper extends the considerations in [7] with an accurate investigation of the effects of the termination impedance on the transmitted power and efficiency as a function of frequency and receiver position. Moreover, efficiency peaks are discussed in this paper. Finally, a numerical characterisation is also shown in order to predict with good accuracy the performance of the system analysed.

This paper is based on magneto-inductive wave theory (MIW) to simply explain the behaviour of a metamaterial. Hence, a brief theoretical discussion of MIW is presented in Section 2. Further, the theoretical modelling of the single resonator used in experiments and the system is shown in Section 3. In Sections 4 and 5, the experimental apparatus and the main results of the transmission coefficient and efficiency are presented, and some comparisons between measures and simulated predictions are given in order to validate and support the experimental tests. Finally, the discussions of the results and conclusions are presented in Section 6.

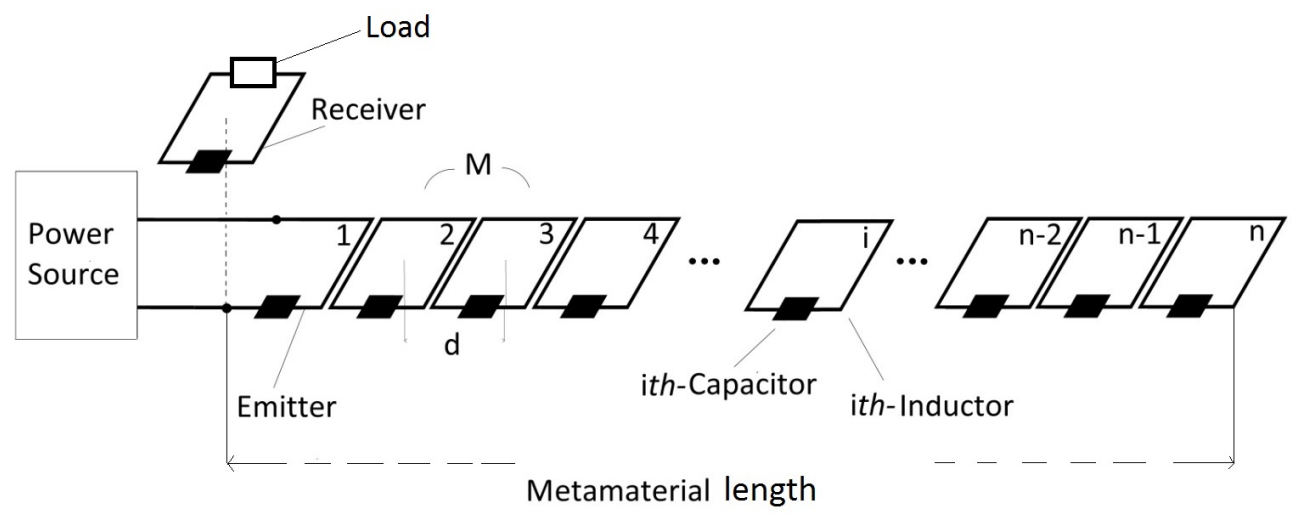

Figure 1. Metamaterial WPT system composed of n-coupled resonators arranged in a line. 


\section{Theoretical Background and Basic Assumptions}

The theory behind magneto-inductive waves (MIW) has been widely discussed in the literature [8-10]. In this section, the main considerations for wireless power transfer applications and equivalent circuits are reviewed. Generally, a metamaterial is a periodic array formed by resonator cells (resonant circuits formed by an inductor $\mathrm{L}$ and a capacitor $\mathrm{C}$ in series) coupled to each other magnetically. Considering a system composed of a finite number $n$ of cells, as shown in Figure 2, the associated equivalent circuit is analytically described by the following system of $n$ equations:

$$
\begin{array}{cc}
\hat{V}_{s}+R_{s} \hat{I}_{1}+\hat{Z} \hat{I}_{1}+j \omega M_{1,2} \hat{I}_{2} & =0 \\
j \omega M_{2,1} \hat{I}_{1}+\hat{Z} \hat{I}_{2}+j \omega M_{2,3} \hat{I}_{3} & =0 \\
\vdots & \vdots \\
j \omega M_{i, i-1} \hat{I}_{i-1}+\hat{Z} \hat{I}_{i}+j \omega M_{i, i+1} \hat{I}_{i+1} & =0 \\
\vdots & \vdots \\
j \omega M_{n, n-1} \hat{I}_{n-1}+\hat{Z} \hat{I}_{n}+\hat{Z}_{T} \hat{I}_{n} & =0
\end{array}
$$

where $\hat{V}_{s}$ and $R_{S}$ are the voltage source applied to the first inductor and its internal resistance, respectively, $\hat{I}_{i}$ the current flowing in the i-th cell, $\hat{Z}=R+j \omega L+1 / j \omega C$ is the impedance of each cell, $R$ is the alternating current (AC) resistance, $L$ the self-inductance, $C$ the capacitance and $\hat{Z}_{T}$ is an optional termination impedance connected to the last cell of the line. Hence, each cell is a L-C series resonant circuit tuned to a single resonant frequency $f_{0}=1 /(2 \pi \sqrt{L C}) . \quad M_{i, i+1}=M_{i+1, i}=M$ is the mutual inductance between two adjacent resonators. In the equivalent circuit, the mutual inductance between two non-adjacent resonators is neglected. According to the MIW theory, the wave travelling along the metamaterial is expressed in terms of current as $\hat{I}_{i}=\hat{I}_{1} e^{-\hat{\gamma}(i-1) d}$, where $\hat{I}_{1}$ is the phasor of the current flowing in the first cell, $d$ is the periodic distance between two adjacent cells and $\hat{\gamma}$ is the propagation constant defined as $\hat{\gamma}=\alpha+j \beta$. $\alpha$ and $\beta$ are the attenuation and phase constants, respectively, and they could also be expressed in terms of electrical parameters; in particular, it is important to express the attenuation per cell, because it represents the wave reduction along the metamaterial:

$$
\alpha d=\sinh ^{-1}\left(\frac{1}{k Q}\right)
$$

where $k=2 M / L$ is the coupling coefficient and $Q=\omega_{0} L / R$ is the quality factor of each inductor. From the coupling coefficient, it is possible to obtain the bandwidth in which the wave propagation is achieved with very low losses, $f_{0} / \sqrt{1+k} \leq f \leq f_{0} / \sqrt{1-k}[11,12]$.

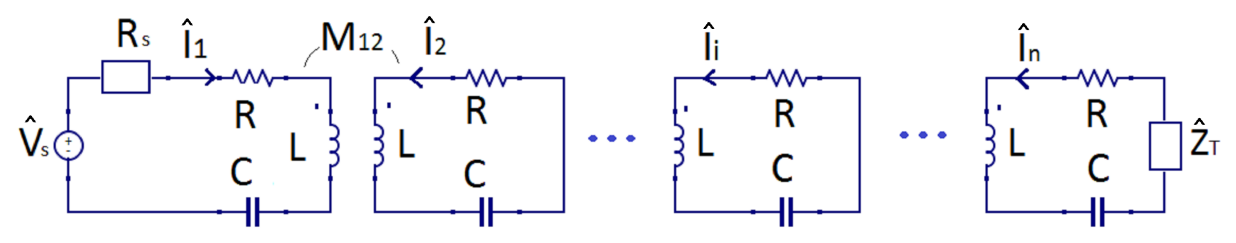

Figure 2. Equivalent circuit of a system composed of $n$ resonator cells with optional termination impedance in the last cell. 
It is known in the literature that the matching condition is achieved by introducing a terminal impedance in the last cell equal to $\hat{Z}_{T}=j \omega M e^{-\hat{\gamma} d}$ (shown in Figure 2), becoming purely real for $f=f_{0}$ and equal to $Z_{T}=\omega_{0} M$, as demonstrated in [11,13].

Generally, considering a receiver inductor with a load $R_{\text {load }}$ in series and facing the $\mathrm{i}$-th cell of the metamaterial, it is enough to add a further equation in Equation (1) in order to represent the whole system shown in Figure 3a:

$$
\begin{array}{cc}
\hat{V}_{s}+R_{s} \hat{I}_{1}+\hat{Z} \hat{I}_{1}+j \omega M \hat{I}_{2} & =0 \\
j \omega M \hat{I}_{1}+\hat{Z} \hat{I}_{2}+j \omega M \hat{I}_{3} & =0 \\
\vdots & \vdots \\
j \omega M \hat{I}_{i-1}+\hat{Z} \hat{I}_{i}+j \omega M \hat{I}_{i+1}+j \omega M_{i, r} \hat{I}_{r} & =0 \\
\vdots & \vdots \\
j \omega M \hat{I}_{n-1}+\hat{Z} \hat{I}_{n}+\hat{Z}_{T} \hat{I}_{n} & =0 \\
j \omega M_{r, i} \hat{I}_{i}+\hat{Z}_{r} \hat{I}_{r} & =0
\end{array}
$$

where $\hat{Z}_{r}=\hat{Z}+R_{\text {load }}$ with the subscript $r$ identifying the receiver inductor. The receiver impedance seen from the nearest cell can be expressed as an impedance of value $\hat{Z}_{d}=\omega^{2} M_{r, i}^{2} / \hat{Z}_{r}$ connected in series to the cell (see Figure 3b). This value is obtained by introducing the expression of $\hat{I}_{r}$, obtained from the last equation of system Equation (3) into the equation of the i-th cell, which can be written:

$$
j \omega M \hat{I}_{i-1}+\left(\hat{Z}+\hat{Z}_{d}\right) \hat{I}_{i}+j \omega M \hat{I}_{i+1}=0 .
$$

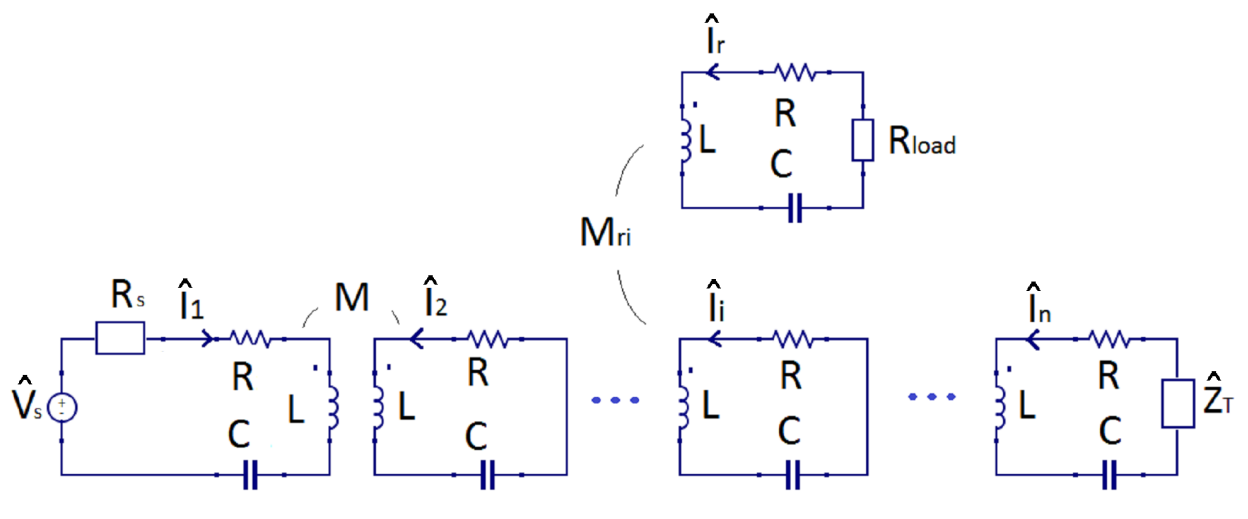

(a)

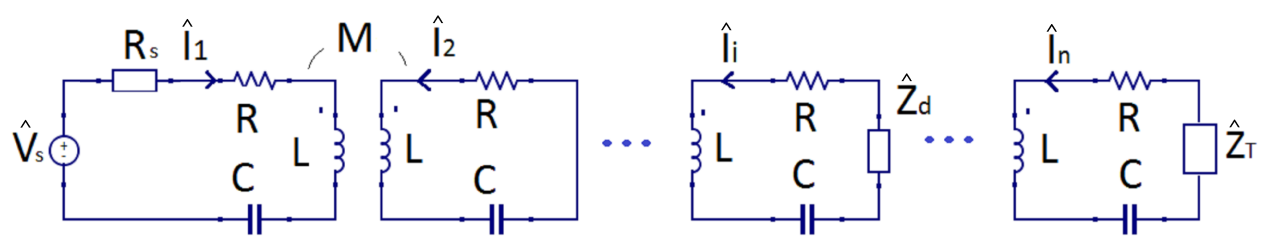

(b)

Figure 3. Equivalent circuit of a system composed of $n$ resonator cells with a receiver (a) and with the impedance $Z_{d}(\mathbf{b})$. 
In this sense, it is enough to set:

$$
\hat{Z}_{d}=\omega_{0} M
$$

in order to obtain the matching condition when the receiver couples to the last cell and avoid a standing wave; this condition can be achieved, for example, by an accurate adjustment of the inductances of the receiver, being $M_{r, i}=k_{r, i} L$ [13]. However, this does not hold if the receiver inductor moves along the metamaterial as a reflected wave is generated, and standing waves result in an extra frequency dependence to the input impedance.

The multiple resonator system can be simplified with an equivalent impedance $\hat{Z}_{e q}$, representing the whole metamaterial and connected in series to the impedance of the first cell, as depicted in Figure 4. If the matching condition is satisfied, then $\hat{Z}_{e q}=\omega_{0} M$ [14]. In all other cases, the equivalent impedance can be also calculated analytically through a continued fraction (as used in [15] for four resonators) whose value depends mainly on the position of the receiver and a possible terminal impedance. For example, considering the receiver located on the third cell of a metamaterial composed of five cells and a termination impedance $\hat{Z}_{T}$, the equivalent impedance can be calculated as follows (this case corresponds to the experimental setup detailed in Section 4):

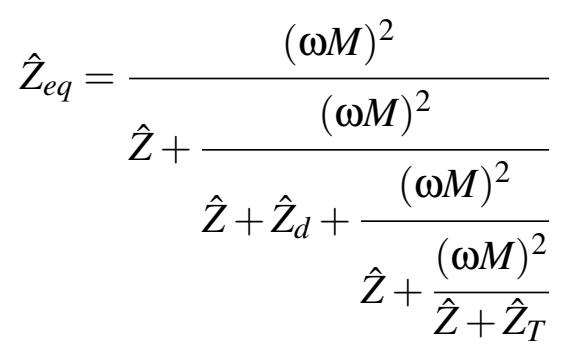

and for $f=f_{0}$ :

$$
\hat{Z}_{e q}=\frac{\left(\omega_{0} M\right)^{2}}{R+\frac{\left(\omega_{0} M\right)^{2}}{R+Z_{d}+\frac{\left(\omega_{0} M\right)^{2}}{R+\frac{\left(\omega_{0} M\right)^{2}}{R+\hat{Z}_{T}}}}}
$$

Hence, the input impedance can be calculated as $\hat{Z}_{i n}=R+\hat{Z}_{e q}$ at the resonant frequency.

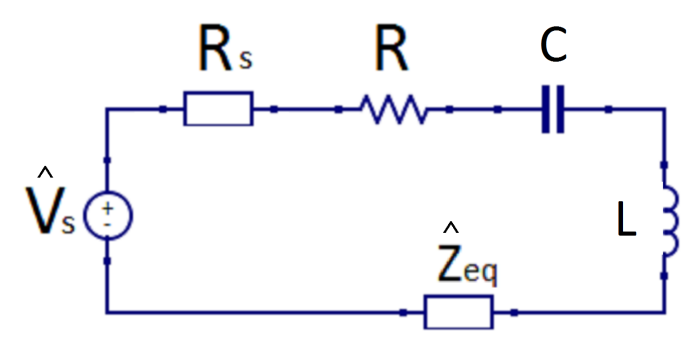

Figure 4. Equivalent circuit of the WPT in terms of an equivalent impedance $\hat{Z}_{e q}$. 


\section{Single Cell and System Characterisation}

In Figure 5, an example of the resonator cell considered in this work and its equivalent lumped circuit model are shown. $L$ and $R$ are respectively the self-inductance and the resistance considered as a function of frequency associated with the metallic track, and $C_{p}$ represents the stray capacitance of the resonator. For a single layer resonator, the self-inductance depends on the external dimension, $l$, the number of turns, $N$, the width of the conductor tracks, $w$, and the space between turns, $s$. The self-inductance $L$ can be predicted with a procedure based on the theory of partial inductance [16]. Here, $L$ is found by calculating the partial self- and mutual inductances between all of the sub-elements in which the whole structure is divided. For the partial self-inductance calculation, the exact formula of rectangular bars proposed in [17] is used, and the numerical procedure detailed in [18] is implemented for the partial mutual inductance calculation. Finally, for the calculation of the stray capacitance and AC resistance, the validated formulas reported in $[19,20]$ can be used in order to take account of both skin and proximity effects increasing significantly with frequency.

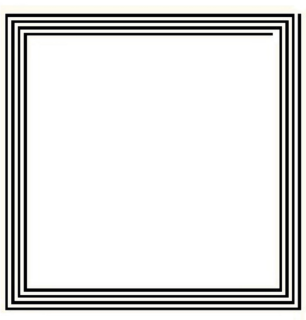

(a)

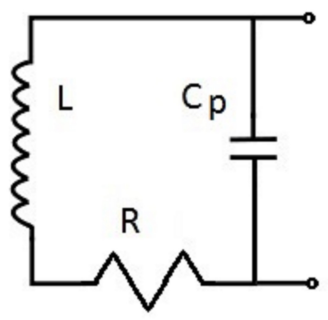

(b)

Figure 5. Example of a spiral resonator considered in this study (a) and its quasi-static equivalent circuit (b).

For a multiple resonator system, the equivalent circuit may be more complex, because the mutual inductance between each couple of cells must be taken into account. Hence, the whole system could be easily represented in a matrix form through the following equation:

$$
\hat{\mathbf{V}}=\hat{\mathbf{Z}} \hat{\mathbf{I}}
$$

where $\hat{\mathbf{V}}=\left[\begin{array}{llll}\hat{V}_{s} & 0 & \ldots & 0\end{array}\right]^{\mathrm{T}}$ with $\hat{V}_{s}$ the phasor supply voltage of the emitter coil, $\hat{\mathbf{I}}$ the phasor of the current flowing in the cells and $\hat{\mathbf{Z}}$ the symmetric matrix of the impedances defined for an $n$ cell system as follows:

$$
\hat{\mathbf{Z}}=\left[\begin{array}{ccccc}
R_{s}+\hat{Z}_{1} & j \omega M_{12} & \ldots & j \omega M_{1 n} & j \omega M_{1 r} \\
j \omega M_{21} & \hat{Z}_{2} & \ldots & j \omega M_{2 n} & j \omega M_{2 r} \\
\vdots & \vdots & \ddots & \vdots & \vdots \\
j \omega M_{n 1} & j \omega M_{n 2} & \ldots & \hat{Z}_{n} & j \omega M_{n r} \\
j \omega M_{r 1} & j \omega M_{r 2} & \ldots & j \omega M_{r n} & \hat{Z}_{r}
\end{array}\right]
$$

where $\hat{Z}_{i}=R_{i}+j \omega L_{i}+1 / j \omega C_{i}(i=1,2, \ldots n)$ represents the impedance of each inductor. In particular, $M_{i j}$ is the mutual inductance between the cells $i$ and $j$ that can be obtained with the same formula used 
for the partial mutual inductance calculation. $C_{i}$ is the total capacitance in series to the self-inductance of each cell considering both the stray, $C_{p}$, and the lumped capacitances added, $C_{a d d}$, to tune the resonance condition $\left(\omega_{0}=1 / \sqrt{L\left(C_{p}+C_{a d d}\right)}\right)$. In this form, the currents circulating in each resonator are calculated by inverting the impedance matrix:

$$
\hat{\mathbf{I}}=\hat{\mathbf{Z}}^{-\mathbf{1}} \hat{\mathbf{V}}
$$

so that the transmitted power and system performance can be quickly numerically predicted [21].

\section{Experimental Setup}

The metamaterial used in this work consists of five equal resonators of a square shape, mutually coupled and arranged in a planar line separated by gaps equal to $g=0.2 \mathrm{~mm}$. The experimental system is shown in Figure 6.

The single metamaterial cell is fabricated with a printed circuit board (PCB) with $N=1, l=40 \mathrm{~mm}$, $w=1 \mathrm{~mm}$ and $s=1 \mathrm{~mm}$. The value of the measured self-inductance is $0.135 \mu \mathrm{H}$, equal to the numerically calculated one. The mutual inductance between two cells is calculated from measurement by resonance splitting, and it is found equal to $-16.3 \mathrm{nH}$ (very close to the predicted one $-18.7 \mathrm{nH}$ ). As the stray capacitance is small, a lumped capacitance equal to $1 \mathrm{nF}$ is added to each cell in order to tune the resonant frequency to $f_{0}=13.56 \mathrm{MHz}$ (as it is a license-free industrial, scientifical and medical (ISM) band). All of the capacitances are parallel to the inductors. The source is connected in series as in Figure 2. Finally, the measured and calculated AC resistances are equal to $0.303 \Omega$ and $0.152 \Omega$, respectively. The measured AC resistance includes also the solder connections and series resistance of the lumped capacitances plus any radiation losses. The layout of the receiver resonator used in these tests is identical to the cells of the metamaterial: a load resistance $\left(R_{\text {load }}=3.3 \Omega\right)$ is soldered in series to the lumped capacitance of $1 \mathrm{nF}$. In case the emitter and receiver coils are single-layer solenoid or single-layer spiral coils, they can be designed with the procedure presented in [22].

An HP 8753 vector network analyser (VNA) (Keysight Technologies, Santa Rosa, CA, USA) was used in order to supply the source and to measure the transmission coefficient $S_{21}$ : the instrument output is connected to the first cell, and the received signal is measured across the load resistance. This type of circuital connection involves an adjustment of the transmitted power measured as the internal impedance of the VNA is equal to $Z_{0}=50 \Omega$, quite different from $R_{\text {load }}$. Hence, the measured value is smaller than the real one; consequently, it is possible to adjust the measure and to find the effective efficiency across the load through:

$$
\eta=\frac{S_{21}^{2}\left(1+Z_{0} / R_{\text {load }}\right)}{1-S_{11}^{2}} .
$$

The transmission coefficient and efficiency are studied for three different conditions, corresponding to different termination impedance values. In particular:

- Condition A: $Z_{T}=0 \Omega$ (corresponding to a short-circuited last cell);

- Condition B: $Z_{T}=120 \Omega$ (much larger than $\left.\omega_{0} M\right)$;

- Condition $\mathrm{C}: Z_{T} \gg 120 \Omega$ (corresponding to an open circuit last cell). 
Finally, the measurements are made considering the receiver inductor sweeping along the whole length of the metamaterial at a height of almost $9 \mathrm{~mm}$, corresponding to the receiver distance at which the matching condition Equation (5) is satisfied.

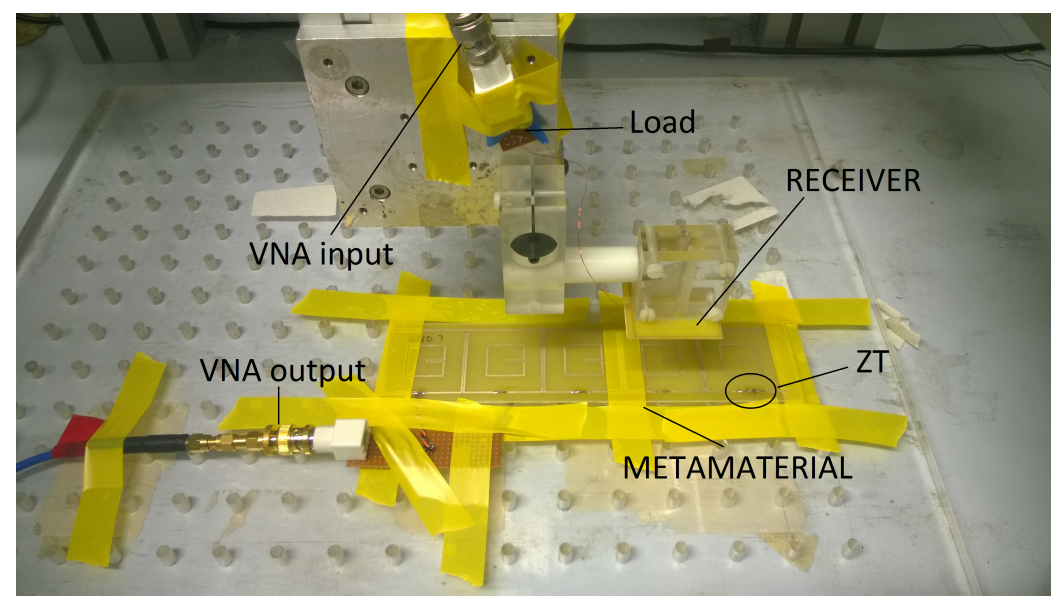

Figure 6. Experimental setup.

\section{Simulation and Measurement Results}

The measured values of the transmission coefficient, $S_{21}$, in $\mathrm{dB}$ for each termination impedance considered as a function of frequency and receiver position are shown in Figure 7. As can be noticed, the plot of $S_{21}$ regarding Condition A is quite different from those relevant to the other two conditions, which are very similar. The maximum values of the transmission coefficient are distributed for different frequencies and positions depending on the considered condition. Particularly, in the frequency range between about 12.4 and $15.5 \mathrm{MHz}$ and when the receiver is positioned at $180 \mathrm{~mm}$, i.e., perfectly facing the last resonator, $S_{21}$ is over $-10 \mathrm{~dB}$ for Condition A, but much smaller for Conditions B and C $(-20 \mathrm{~dB})$. Moreover, for Condition A, the concentration of high transmission coefficient values around the resonant frequency means that the matching condition is achieved. For Conditions $\mathrm{B}$ and $\mathrm{C}$, high transmission coefficient values occur for different positions of the receiver along the metamaterial, as is confirmed by the measures of $S_{21}$ and the adjusted efficiency presented in Figure 8.

In fact, as can be seen, the behaviour of the efficiency alternates highs and lows, and the peaks are located every two cells. If the last cell is short-circuited $\left(Z_{T}=0 \Omega\right)$, the maximum of the efficiency is achieved when the receiver faces the I, III and V resonator (cell centres at 20, 100 and $180 \mathrm{~mm}$ ). The introduction of a high impedance $\left(Z_{T}=120 \Omega\right.$ or $\left.Z_{T} \gg 120 \Omega\right)$ involves a displacement of peaks by one cell: in fact, the efficiency peaks occur when the receiver is on the II and IV cell (cell centres at 60 and $140 \mathrm{~mm}$ ). Furthermore, observing the equivalent impedance values reported in Table 1, the value of $Z_{e q}$ is very close to $\omega_{0} M=1.39 \Omega$ in positions where the efficiency is maximum. Finally, the correspondence of the peaks at the resonant frequency is further validated by showing the behaviour of $S_{21}$ as a function of frequency when the receiver is perfectly located on each cell, as depicted in Figure 9. The behaviour of the transmission coefficient is strongly affected by the introduction of a termination impedance $Z_{T}$, which changes the distribution of the transmission coefficient peaks in the resonant frequency bandwidth. In particular, with the connection or disconnection of the termination impedance, the maximum values of $S_{21}$ can be obtained at the resonant frequency in all of the receiver positions. 


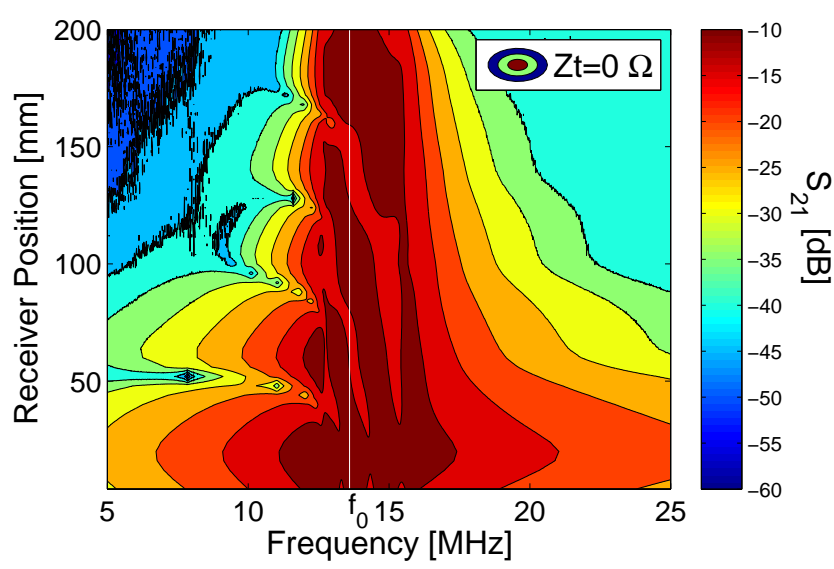

(a)

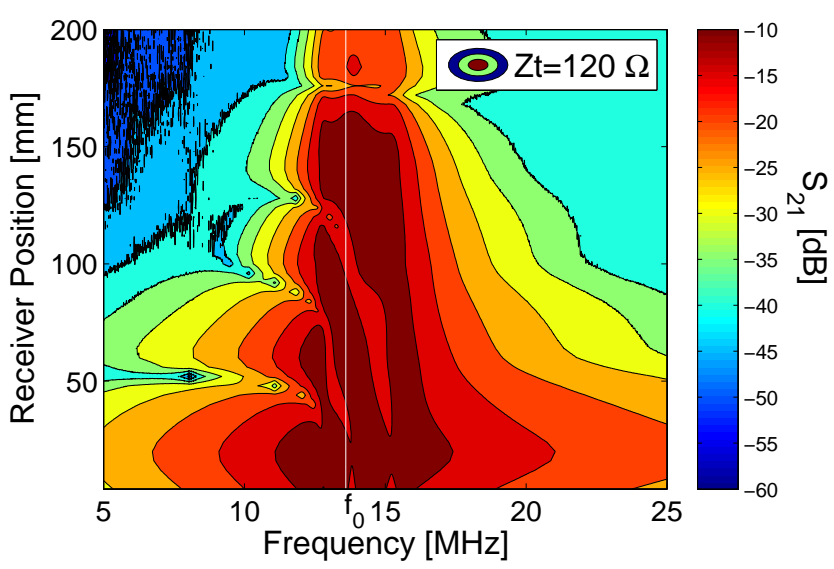

(b)

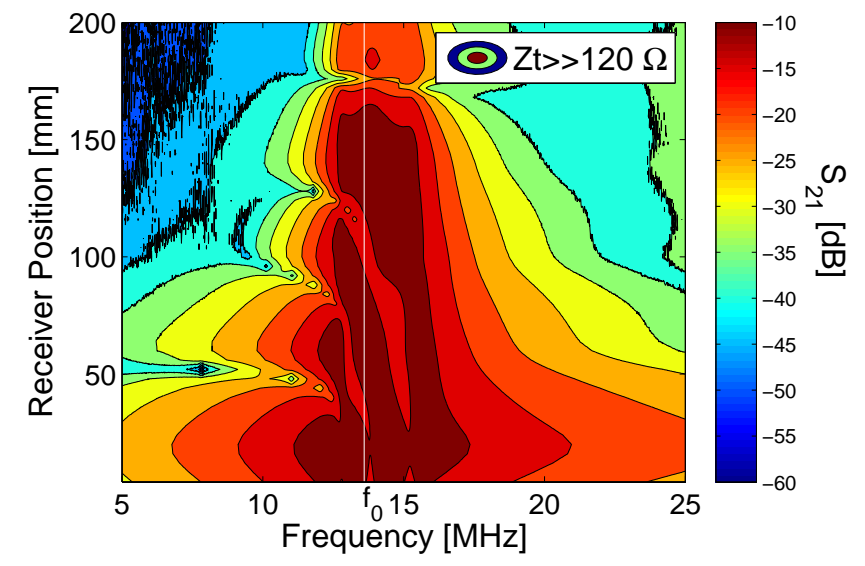

(c)

Figure 7. Measurements of $S_{21}$ as a function of frequency and receiver position for: (a) $Z_{T}=0 \Omega$; (b) $Z_{T}=120 \Omega$; and (c) $Z_{T} \gg 120 \Omega$.

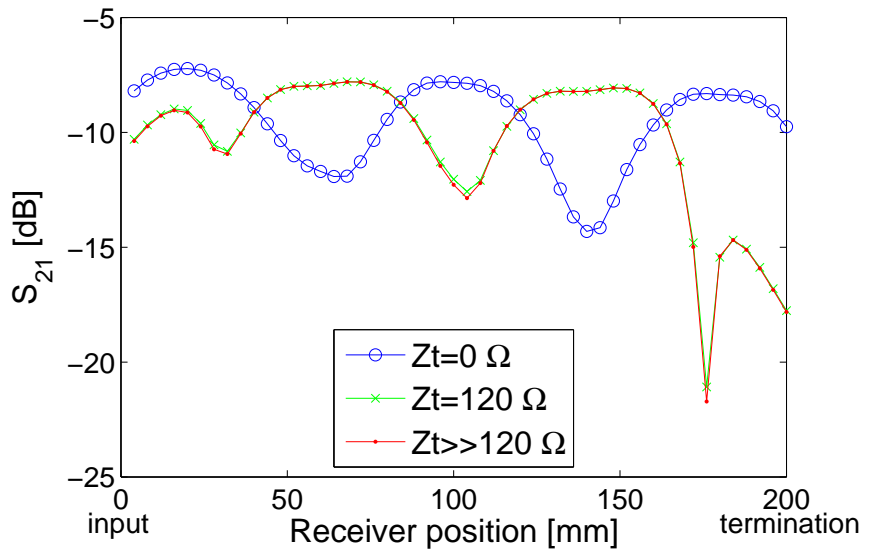

(a)

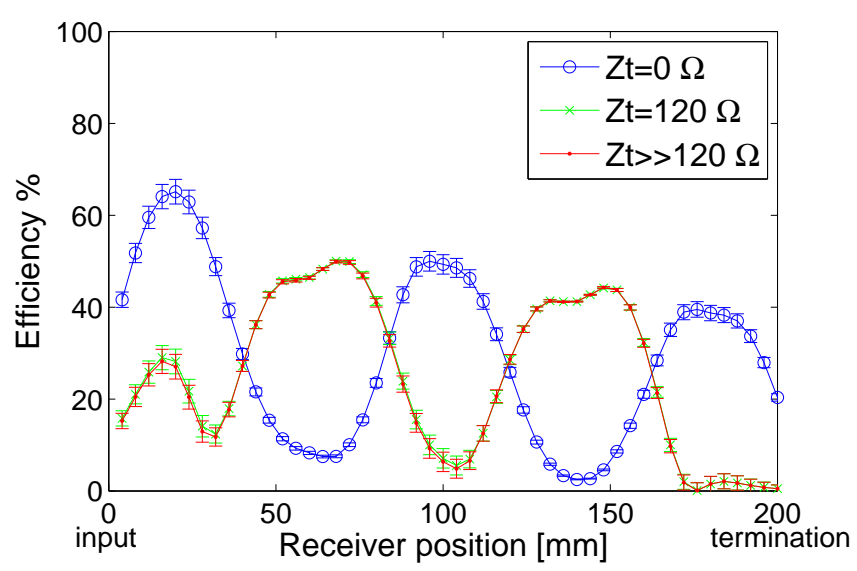

(b)

Figure 8. Measurements of $S_{21}$ (a) and adjusted efficiency (b) as a function of the receiver position for the termination impedances used and at $f=f_{0}$. 


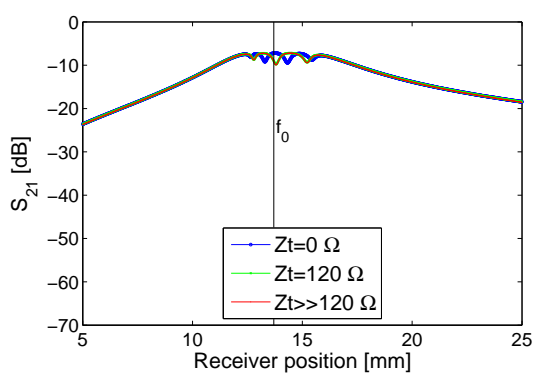

(a)

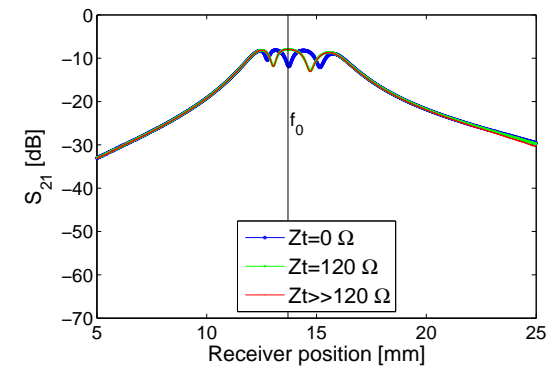

(b)

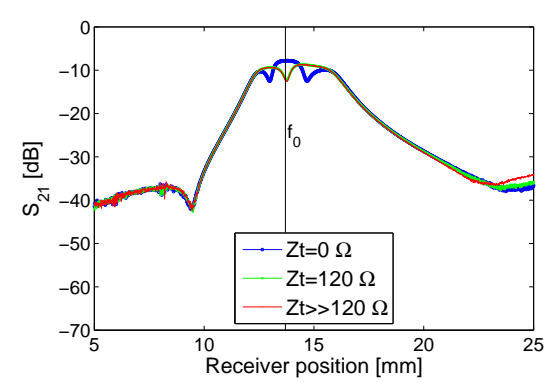

(c)

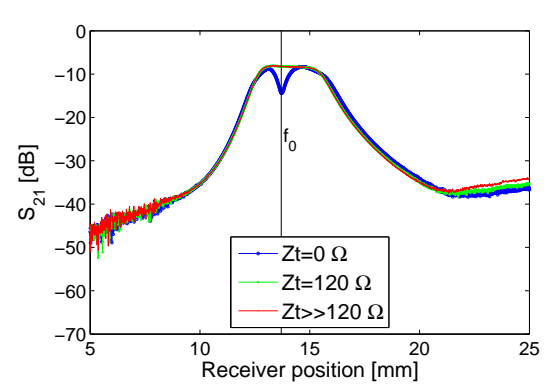

(d)

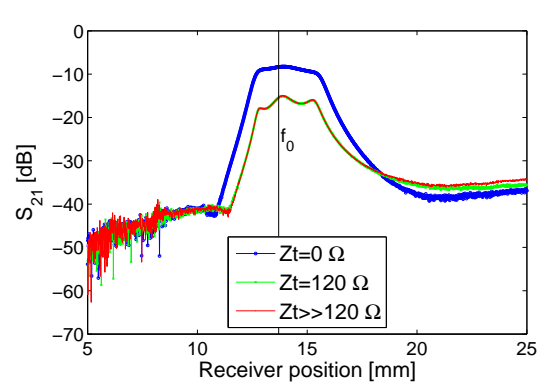

(e)

Figure 9. $S_{21}$ measured as a function of frequency for all receiver positions facing the I (a), II (b), III (c), IV (d) and V (e) cell.

Table 1. Equivalent impedance values, $Z_{\text {eq }}$, and efficiency, $\eta$, at $f=f_{0}$ for different receiver positions and conditions calculated with Equation (7).

\begin{tabular}{ccccc}
\hline Receiver position & \multicolumn{2}{c}{$Z_{T}=0 \Omega$} & \multicolumn{2}{c}{$Z_{T}=120 \Omega$} \\
& & \multicolumn{3}{c}{$Z_{T} \gg 120 \Omega$} \\
\cline { 2 - 5 } & $Z_{e q}[\Omega]$ & $\eta \%$ & $Z_{e q}[\Omega]$ & $\eta \%$ \\
\hline I cell & 1.6 & $67 \%$ & 7.2 & $30 \%$ \\
II cell & 0.2 & $10 \%$ & 1.1 & $58 \%$ \\
III cell & 1.4 & $53 \%$ & 6.2 & $5 \%$ \\
IV cell & 0.3 & $5 \%$ & 1.2 & $49 \%$ \\
V cell & 1.3 & $43 \%$ & 5.8 & $0 \%$ \\
\hline
\end{tabular}

The measurements are then compared with numerical simulations, made implementing the formulas in Section 3 in a MATLAB programme [23]. In particular, the efficiency, peaks of efficiency and optimum frequencies are studied, taking into account only two different conditions, $\mathrm{A}\left(Z_{T}=0 \Omega\right.$, shown in Figure 10a,c) and B $\left(Z_{T}=120 \Omega\right.$, shown in Figure $\left.10 \mathrm{~b}, \mathrm{~d}\right)$, as Conditions B and $\mathrm{C}\left(Z_{T} \gg 120 \Omega\right)$ are found to be equivalent. Peaks of efficiency are defined as the maximum values of efficiency occurring at the resonant frequency and optimum frequencies as those frequencies at which the peaks of efficiency are achieved. As shown, all of the simulations match with very good agreement the experimental data. Considering the efficiency, the largest difference is obtained for the receiver centre between 0 and 60 $\mathrm{mm}$ in Condition B (as can be noticed in Figure 10b). Moreover, a small difference (10\%) is obtained for the receiver centre between $80 \mathrm{~mm}$ and $120 \mathrm{~mm}$, regarding the peaks of efficiency. Consequently, the 
prediction of the optimum frequencies shows a little disagreement with the measurement for the same receiver positions (as shown in Figure 10d), even if the general behaviour is similar to the measurement.

Finally, it is worth noticing that the efficiency and efficiency peaks are very close when the receiver is perfectly aligned with Resonators I, III and V for Condition A (see Figure 10a) or to Resonators II and IV for Condition B (see Figure 10b). Correspondingly, in the same positions, the optimum frequencies of both Conditions $\mathrm{A}$ and $\mathrm{B}$ are close to the resonant frequency, as shown in Figure 10b,d, respectively. This behaviour enhances the assumption that the matching condition occurs every two cells (as previously demonstrated by the equivalent impedance values; Table 1).

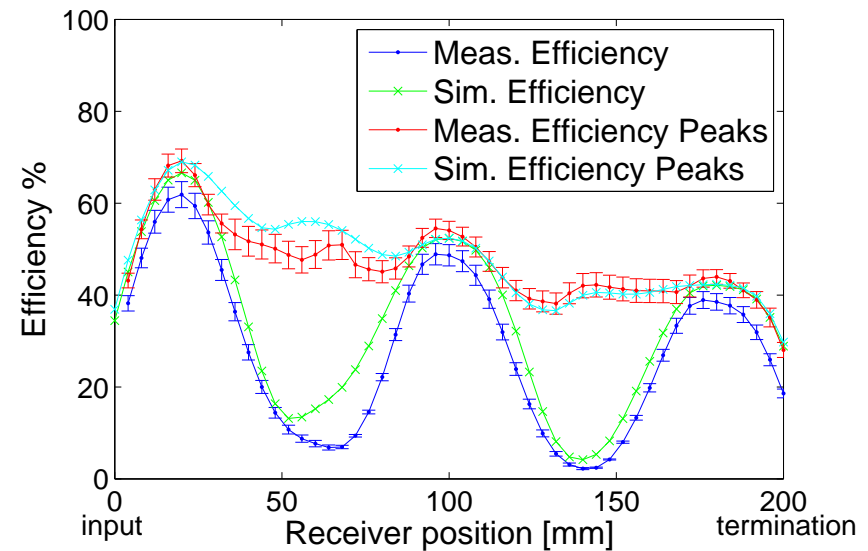

(a)

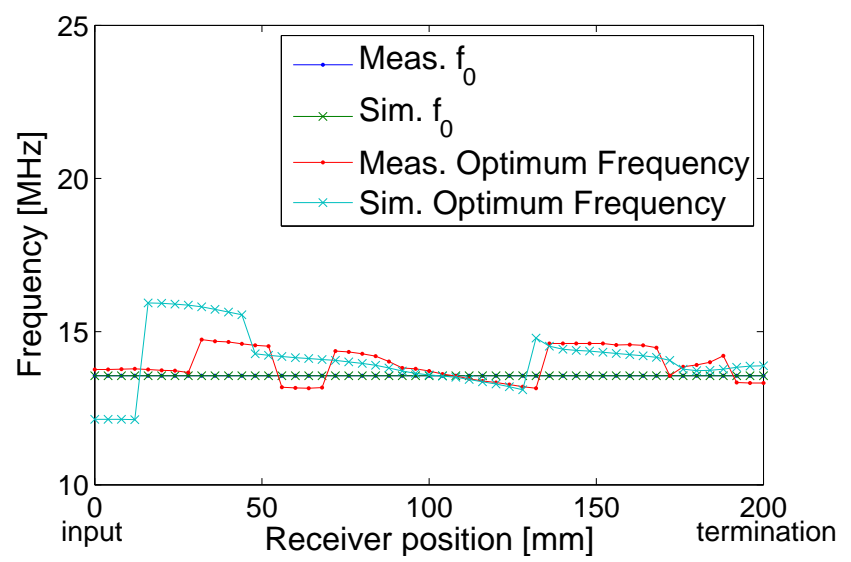

(c)

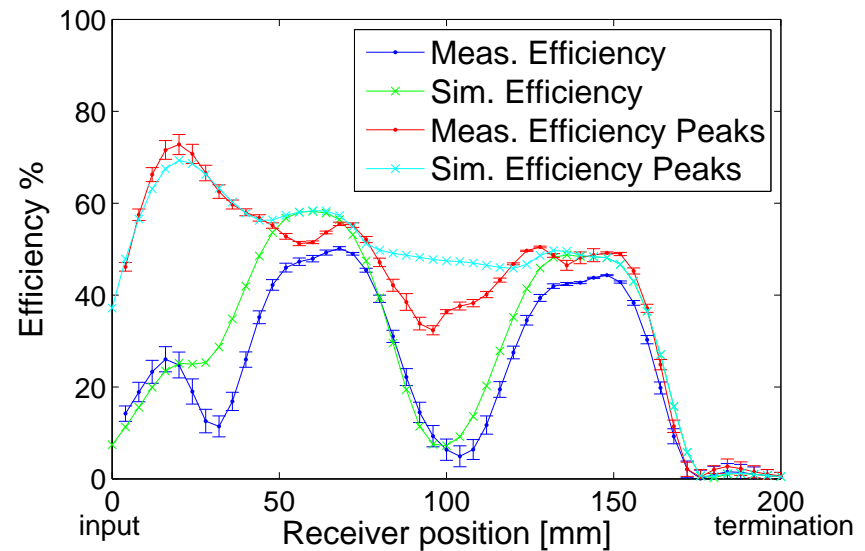

(b)

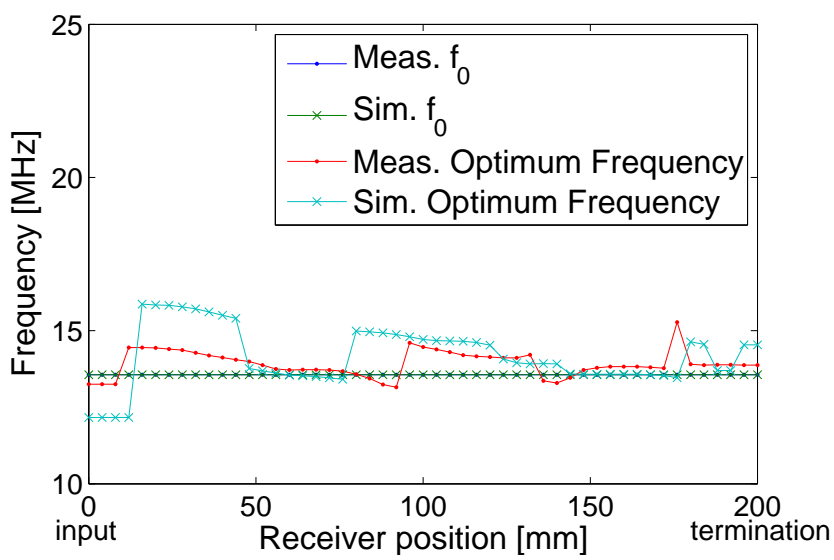

(d)

Figure 10. Comparison of measured and simulated efficiency and efficiency peaks and optimum frequency for $Z_{T}=0 \Omega$ (a) and (c) and $Z_{T}=120 \Omega$, (b) and (d), respectively.

\section{Discussion and Conclusions}

The experimental analysis presented in this work shows the behaviour of the power delivered to a receiver sliding along a metamaterial for three different values of a termination impedance, $Z_{T}$ (Conditions A, B and C presented in Section 4). In the experiments, it was found that the termination impedance affects the received power behaviour modifying the equivalent impedance $Z_{e q}$ and, hence, the position of the receiver where the maximum value of efficiency is achieved. In this way, it is possible to 
obtain the best behaviour for any position of the receiver by varying the $Z_{T}$ value between zero and a high value (in this work, $120 \Omega$, almost a hundred-times larger than $\omega_{0} M$ ) in order to maintain the equivalent impedance at always about $\omega_{0} M$ (as reported in Table 1). In Figure 11, the envelopes of the efficiency and peaks of efficiency obtained for the termination impedances of 0 and $120 \Omega$ with the respective optimum frequencies are shown as a function of the receiver position. It can be seen that the envelope of the efficiency has globally higher values than the cases with constant termination impedances. Moreover, the envelope of the efficiency overlaps the envelope of the efficiency peaks for a number of receiver positions. Furthermore, the optimum frequencies, with the exception of the first cells, are closer to the resonant frequency: this is due to the equivalent impedance closer to the matching condition.

Transmitted power and efficiency are investigated under different termination impedance conditions, which improve significantly the efficiency at the resonant frequency. All of the considerations presented in this work are supported by circuital, numerical and experimental results.

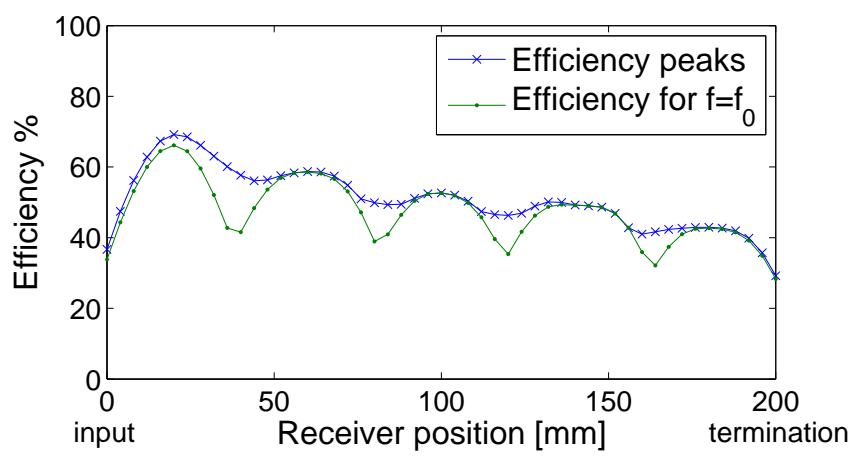

(a)

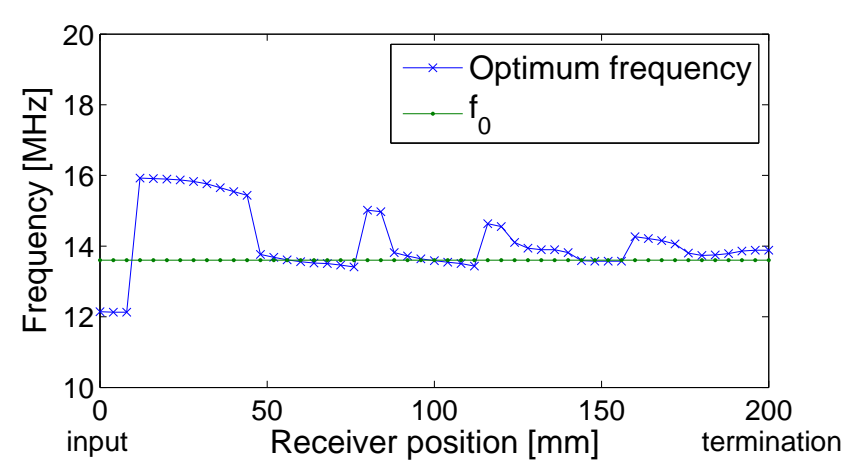

(b)

Figure 11. Comparison between simulated envelopes of efficiency and efficiency peaks (a) and optimum frequencies (b).

\section{Author Contributions}

G. Puccetti performed the simulations and wrote the paper. C. J. Stevens gave input to the theoretical work, organized the experimental setup and measurements. U. Reggiani and L. Sandrolini contributed to the writing and organization of the paper with particular reference to the presentation and discussion of the simulation and measurement results. 


\section{Conflicts of Interest}

The authors declare no conflict of interest.

\section{References}

1. Villa, J.L.; Sallán, J.; Llombart, A.; Sanz, J.F. Design of a high frequency Inductively coupled power transfer system for electric vehicle battery charge. Appl. Energy 2009, 86, 355-363.

2. Jang, Y.; Jovanovic, M. A contactless electrical energy transmission system for portable-telephone battery chargers. IEEE Trans. Ind. Electron. 2003, 50, 520-527.

3. RamRakhyani, A.; Mirabbasi, S.; Chiao, M. Design and optimization of resonance-based efficient wireless power delivery systems for biomedical implants. IEEE Trans. Biomed. Circuits Syst. 2011, 5, 48-63.

4. Wang, B.; Yerazunis, W.; Teo, K.H. Wireless power transfer: Metamaterials and array of coupled resonators. Proc. IEEE 2013, 101, 1359-1368.

5. Urzhumov, Y.; Smith, D.R. Metamaterial-enhanced coupling between magnetic dipoles for efficient wireless power transfer. Phys. Rev. B 2011, 83, doi:10.1103/PhysRevB.83.205114.

6. Kim, H.; Seo, C. Highly efficient wireless power transfer using metamaterial slab with zero refractive property. Electron. Lett. 2014, 50, 1158-1160.

7. Stevens, C. Magnetoinductive waves and wireless power transfer. IEEE Trans. Power Electron. 2014, $P P, 1$.

8. Shamonina, E.; Kalinin, V.; Ringhofer, K.; Solymar, L. Magneto-inductive waveguide. Electron. Lett. 2002, 38, 371-373.

9. Chan, C.; Stevens, C. Two-dimensional magneto-inductive wave data structures. In Proceedings of 5th European Conference on Antennas and Propagation (EUCAP), Rome, Italy, 11-15 April 2011; pp. 1071-1075.

10. Shamonina, E.; Kalinin, V.A.; Ringhofer, K.H.; Solymar, L. Magnetoinductive waves in one, two, and three dimensions. J. Appl. Phys. 2002, 92, 6252-6261.

11. Solymar, L.; Shamonina, E. Waves in Metamaterials; Oxford University Press (OUP): Oxford, UK, 2009.

12. Stevens, C.; Chan, C.; Stamatis, K.; Edwards, D. Magnetic Metamaterials as 1-D Data Transfer Channels: An Application for Magneto-Inductive Waves. IEEE Trans. Microw. Theory Tech. 2010, 58, 1248-1256.

13. Syms, R.R.A.; Young, I.R.; Solymar, L. Low-loss magneto-inductive waveguides. J. Phys. D Appl. Phys. 2006, 39, 3945, doi:10.1088/0022-3727/39/18/004.

14. Stevens, C. Power transfer via metamaterials. Comput. Mater. Contin. 2013, 33, 1-18.

15. Zhang, Y.; Zhao, Z.; Chen, K. Frequency-splitting analysis of four-coil resonant wireless power transfer. IEEE Trans. Ind. Appl. 2014, 50, 2436-2445.

16. Ruehli, A.E. Inductance calculations in a complex integrated circuit environment. IBM J. Res. Dev. 1972, 16, 470-481.

17. Hoer, C.; Love, C. Exact inductance equations for rectangular conductors with applications to more complicated geometries. J. Res. Nat. Bur. Stand. Sec. C Eng. Inst. 1965, 69C, 127-137. 
18. Sonntag, C.; Lomonova, E.; Duarte, J. Implementation of the Neumann formula for calculating the mutual inductance between planar PCB inductors. In Proceedings of 18th International Conference on Electrical Machines, Vilamoura, Portugal, 6-9 September 2008; pp. 1-6.

19. Bilotti, F.; Toscano, A.; Vegni, L. Design of Spiral and Multiple Split-Ring Resonators for the Realization of Miniaturized Metamaterial Samples. IEEE Trans. Antennas Propag. 2007, $55,2258-2267$.

20. Jow, U.M.; Ghovanloo, M. Design and optimization of printed spiral coils for efficient transcutaneous inductive power transmission. IEEE Trans. Biomed. Circuits Syst. 2007, 1, 193-202.

21. Puccetti, G.; Reggiani, U.; Sandrolini, L. Experimental analysis of wireless power transmission with spiral resonators. Energies 2013, 6, 5887-5896.

22. Sandrolini, L.; Reggiani, U.; Puccetti, G.; Neau, Y. Equivalent circuit characterization of resonant magnetic coupling for wireless transmission of electrical energy. Int. J. Circuit Theory Appl. 2013, 41, 753-771.

23. MATLAB. Version 8.0.0 (R2012b); The MathWorks Inc.: Natick, MA, USA, 2012.

(C) 2015 by the authors; licensee MDPI, Basel, Switzerland. This article is an open access article distributed under the terms and conditions of the Creative Commons Attribution license (http://creativecommons.org/licenses/by/4.0/). 